\title{
Ц.Д. Норбосамбуев
}

\section{О СУММАХ ДИАГОНАЛЬНЫХ И ОБРАТИМЫХ ОБОБЩЕННЫХ МАТРИЦ}

\begin{abstract}
Исследованы свойства хороших колец обобщенных матриц. Показано, что любая обобщенная матрица есть сумма диагональной и обратимой обобщенных матриц. Получено одно условие $k$-хорошести произвольного кольца обобщенных матриц.
\end{abstract}

Ключевые слова: кольцо, обобщенная матрица, формальная матрица, k-хорошее кольцо.

\section{1. Введение}

Числовые матрицы используются во многих областях математики и в различных её приложениях. В алгебре часто встречаются и имеют большое значение так называемые обобщенные матрицы. Их называют также формальными матрицами. Элементы этих матриц могут принимать значения в нескольких кольцах и бимодулях. Обобщенные матрицы складываются и умножаются по стандартным правилам матричного сложения и умножения. В результате получается кольцо кольцо обобщенных (или формальных) матриц.

Это кольцо представляет собой важный алгебраический объект. Например, кольцо эндоморфизмов разложимого в прямую сумму модуля и любое кольцо с нетривиальным идемпотентом являются кольцами обобщенных матриц. Кольца обобщенных матриц играют важную роль в изучении ряда классов артиновых колец и алгебр. Исследование колец обобщенных матриц - это актуальное направление в современной теории колец и модулей. Оно имеет большое научное значение. В настоящее время эта тематика привлекает повышенное внимание зарубежных специалистов. С определением и основными свойствами колец обобщенных матриц можно познакомиться в статьях [1-3]. Напомним, что $K_{n}$ обозначает некоторое кольцо обобщенных матриц порядка $n$

$$
K_{n}=\left(\begin{array}{cccc}
R_{1} & M_{12} & \ldots & M_{1 n} \\
M_{21} & R_{2} & \ldots & M_{2 n} \\
\ldots & \ldots & \ldots & \ldots \\
M_{n 1} & M_{n 2} & \ldots & R_{n}
\end{array}\right),
$$

где $R_{1}, \ldots, R_{n}$ - некоторые кольца, $M_{i j}-R_{i}-R_{j}$-бимодуль, $i, j=1, \ldots, n$.

Пусть $k$ - натуральное число, $k \geq 2, R$ - произвольное кольцо. Элемент $a$ кольца $R$ называется $k$-хорошим, если его можно записать в виде суммы $k$ обратимых элементов кольца $R$. Кольцо называется $k$-хорошим, если каждый его элемент является $k$-хорошим. Изучение колец, порождаемых аддитивно своими обратимыми элементами, началось в 1953-1954 годах, когда Вольфсон [4] и Зелинский [5] независимо друг от друга показали, что всякое линейное отображение векторного пространства $V$ над телом $D$ есть сумма двух обратимых линейных отображений, кроме случая, когда $\operatorname{dim}(V)=1$ и $D=Z_{2}$. Это значит, что кольцо линейных преоб- 
разований $\operatorname{End}(V)$ порождается аддитивно своими обратимыми элементами. В 1958 году Скорняков [6] поставил задачу описания такого рода колец. В [7] Рaфаэль, отвечая на вопрос Скорнякова, дал начало систематическому изучению таких колец, которые он назвал $S$-кольцами. Независимо от предыдущих работ к этой проблеме пришел Фукс. В [8] он сформулировал вопрос: «Когда автоморфизмы абелевой группы порождают аддитивно её кольцо эндоморфизмов?» За этим последовал ряд статей Стрингалла [9], Фридмана [10], Хилла [11] и Кастаньо [12]. В 1973 г. Хенриксен [13] описал два широких класса колец, порождаемых своими обратимыми элементами. Позже с этими кольцами работали Вамос [14] (он ввел понятие $k$-хорошего кольца), Сривастава [15]. Имеется несколько статей, посвященных различным $k$-хорошим кольцам. Так, например, в [14] и [15] получены результаты по $k$-хорошести регулярных колец фон Неймана, правых самоинъективных колец.

\section{2. Одно условие $\boldsymbol{k}$-хорошести произвольного кольца обобщенных матриц}

Теорема 1. Кольцо $K_{n}$ является $k$-хорошим, если все $R_{i}-k$-хорошие кольца для некоторого $k>1, i=1, \ldots, n$.

Доказательство. Пусть все $R_{i}-k$-хорошие кольца, $k>1, i=1, \ldots, n$ и $X \in K_{n}$. Запишем матрицу $X$ в полном виде:

$$
X=\left(\begin{array}{cccc}
r_{1} & m_{12} & \ldots & m_{1 n} \\
m_{21} & r_{2} & \ldots & m_{2 n} \\
\ldots & \ldots & \ldots & \ldots \\
m_{n 1} & m_{n 2} & \ldots & r_{n}
\end{array}\right)
$$

Зададим матрицы $A, B$ и $C$ следующим образом:

$$
A=\left(\begin{array}{cccc}
0 & m_{12} & \ldots & m_{1 n} \\
0 & 0 & \ldots & m_{2 n} \\
\ldots & \ldots & \ldots & \ldots \\
0 & 0 & \ldots & 0
\end{array}\right), B=\left(\begin{array}{cccc}
r_{1} & 0 & \ldots & 0 \\
0 & r_{2} & \ldots & 0 \\
\ldots & \ldots & \ldots & \ldots \\
0 & 0 & \ldots & r_{n}
\end{array}\right), C=\left(\begin{array}{cccc}
0 & 0 & \ldots & 0 \\
m_{21} & 0 & \ldots & 0 \\
\ldots & \ldots & \ldots & \ldots \\
m_{n 1} & m_{n 2} & \ldots & 0
\end{array}\right) .
$$

Очевидно, что $X=A+B+C$.

Так как все $R_{i}-k$-хорошие кольца, то

$$
\begin{gathered}
B=\left(\begin{array}{cccc}
r_{1} & 0 & \ldots & 0 \\
0 & r_{2} & \ldots & 0 \\
\ldots & \ldots & \ldots & \ldots \\
0 & 0 & \ldots & r_{n}
\end{array}\right)=\left(\begin{array}{cccc}
u_{1}^{1}+u_{1}^{2}+\ldots+u_{1}^{k} & 0 & \ldots & 0 \\
0 & u_{2}^{1}+u_{2}^{2}+\ldots+u_{2}^{k} & \ldots & 0 \\
\ldots & \ldots & \ldots \\
\ldots & 0 & \ldots & u_{n}^{1}+u_{n}^{2}+\ldots+u_{n}^{k}
\end{array}\right)= \\
=\left(\begin{array}{cccc}
u_{1}^{1} & 0 & \ldots & 0 \\
0 & u_{2}^{1} & \ldots & 0 \\
\ldots & \ldots & \ldots & \ldots \\
0 & 0 & \ldots & u_{n}^{1}
\end{array}\right)+\left(\begin{array}{cccc}
u_{1}^{2} & 0 & \ldots & 0 \\
0 & u_{2}^{2} & \ldots & 0 \\
\ldots & \ldots & \ldots & \ldots \\
0 & 0 & \ldots & u_{n}^{2}
\end{array}\right)+\ldots+\left(\begin{array}{cccc}
u_{1}^{k} & 0 & \ldots & 0 \\
0 & u_{2}^{k} & \ldots & 0 \\
\ldots & \ldots & \ldots & \ldots \\
0 & 0 & \ldots & u_{n}^{k}
\end{array}\right)= \\
=U_{1}+U_{2}+\ldots+U_{k},
\end{gathered}
$$

где $U_{1}, U_{2}, \ldots, U_{k}-$ обратимые матрицы. 
Определим теперь матрицы $A^{\prime}$ и $C^{\prime}$ следующим образом:

$$
\begin{gathered}
A^{\prime}=A+U_{1}=\left(\begin{array}{cccc}
u_{1}^{1} & m_{12} & \ldots & m_{1 n} \\
0 & u_{2}^{1} & \ldots & m_{2 n} \\
\ldots & \ldots & \ldots & \ldots \\
0 & 0 & \ldots & u_{n}^{1}
\end{array}\right), \\
C^{\prime}=C+U_{2}=\left(\begin{array}{cccc}
u_{1}^{2} & 0 & \ldots & 0 \\
m_{21} & u_{2}^{2} & \ldots & 0 \\
\ldots & \ldots & \ldots & \ldots \\
m_{n 1} & m_{n 2} & \ldots & u_{n}^{2}
\end{array}\right) .
\end{gathered}
$$

Легко видеть, что $A^{\prime}$ и $C^{\prime}$ - обратимые матрицы как треугольные матрицы с обратимыми элементами на главной диагонали.

Таким образом, имеем $X=A+B+C=A^{\prime}+C^{\prime}+U_{3}+\ldots U_{k}-$ сумма $k$ обратимых матриц.

Итак, $K_{n}$ является $k$-хорошим кольцом. Что и требовалось доказать.

\section{3. О суммах диагональных и обратимых обобщенных матриц}

Через $\mathrm{M}(n, R)$ будем обозначать обычное кольцо квадратных матриц порядка $n$ над кольцом $R$. Хенриксеном [5] было показано, что $\mathrm{M}(n, R)-3$-хорошее кольцо. А именно, он доказал, что всякая матрица из этого кольца представима в виде суммы диагональной и обратимой матриц. Диагональная же матрица всегда может быть записана как сумма двух обратимых матриц. Кольцо $\mathrm{M}(n, R)$, конечно, является кольцом обобщенных матриц. На главной диагонали матриц из $\mathrm{M}(n, R)$ находятся элементы кольца $R$. На остальных местах - элементы $R-R$-бимодуля $R$. Умножение задается с помощью тождественных гомоморфизмов.

Докажем, что обобщенная матрица также есть сумма диагональной и обратимой обобщенных матриц.

Доказательство следующей леммы элементарно.

Лемма 2. Пусть $R$ - кольцо, $x \in R, p, q \in U(R)$ - множеству обратимых элементов кольца $R$. Если $p \cdot x \cdot q=1$, то $x \in U(R)$ и $x^{-1}=q \cdot p$.

Лемма 3. Пусть $U^{\prime}$ - блочная матрица порядка 2 (обобщенная матрица порядка $n+1)$

$$
U^{\prime}=\left(\begin{array}{cc}
U & B \\
C & 1+C U^{-1} B
\end{array}\right),
$$

где $U$ - обратимая обобщенная матрица порядка $n, B$ - вектор-столбец длины $n, C$ - вектор-строка длины $n$. Тогда $U^{\prime}$ - обратимая матрица и

$$
\left(U^{\prime}\right)^{-1}=\left(\begin{array}{cc}
U^{-1}+(-U)^{-1} B(-C) U^{-1} & (-U)^{-1} B \\
-C U^{-1} & 1
\end{array}\right) .
$$

Доказательство. Положим

$$
P=\left(\begin{array}{cc}
E_{n} & 0 \\
-C U^{-1} & 1
\end{array}\right) \text { и } Q=\left(\begin{array}{cc}
U^{-1} & (-U)^{-1} B \\
0 & 1
\end{array}\right),
$$

где $E_{n}$ - единичная матрица кольца $K_{n}$ обобщенных матриц порядка $n$. Убедимся, что $P$ и $Q$ - обратимые матрицы в кольце $K_{n+1}$. 
Действительно, утверждаем, что

$$
P^{-1}=\left(\begin{array}{cc}
E_{n} & 0 \\
C U^{-1} & 1
\end{array}\right) \text { и } Q^{-1}=\left(\begin{array}{cc}
U & B \\
0 & 1
\end{array}\right) .
$$

Запишем следующие равенства:

$$
\begin{gathered}
P P^{-1}=\left(\begin{array}{cc}
E_{n} & 0 \\
-C U^{-1} & 1
\end{array}\right)\left(\begin{array}{cc}
E_{n} & 0 \\
C U^{-1} & 1
\end{array}\right)= \\
=\left(\begin{array}{cc}
E_{n} * E_{n}+0 *\left(C U^{-1}\right) & E_{n} * 0+0 * 1 \\
\left(-C U^{-1}\right) * E_{n}+1 *\left(C U^{-1}\right) & \left(-C U^{-1}\right)^{*} 0+1 * 1
\end{array}\right)=\left(\begin{array}{cc}
E_{n} & 0 \\
0 & 1
\end{array}\right), \\
P^{-1} P=\left(\begin{array}{cc}
E_{n} & 0 \\
C U^{-1} & 1
\end{array}\right)\left(\begin{array}{cc}
E_{n} & 0 \\
-C U^{-1} & 1
\end{array}\right)= \\
=\left(\begin{array}{cc}
E_{n} * E_{n}+0 *\left(-C U^{-1}\right) & E_{n} * 0+0 * 1 \\
\left(C U^{-1}\right)^{*} E_{n}+1 *\left(-C U^{-1}\right) & \left(C U^{-1}\right) * 0+1 * 1
\end{array}\right)=\left(\begin{array}{cc}
E_{n} & 0 \\
0 & 1
\end{array}\right) .
\end{gathered}
$$

И далее имеем равенства

$$
\begin{gathered}
Q Q^{-1}=\left(\begin{array}{cc}
U^{-1} & (-U)^{-1} B \\
0 & 1
\end{array}\right)\left(\begin{array}{cc}
U & B \\
0 & 1
\end{array}\right)= \\
=\left(\begin{array}{cc}
U^{-1} * U+\left(-U^{-1} B\right) * 0 & U^{-1} * B+\left(-U^{-1} B\right) * 1 \\
0 * U+1 * 0 & 0 * B+1 * 1
\end{array}\right)=\left(\begin{array}{cc}
E_{n} & 0 \\
0 & 1
\end{array}\right), \\
Q^{-1} Q=\left(\begin{array}{cc}
U & B \\
0 & 1
\end{array}\right)\left(\begin{array}{cc}
U^{-1} & (-U)^{-1} B \\
0 & 1
\end{array}\right)= \\
=\left(\begin{array}{cc}
U^{*} U^{-1}+B^{*} 0 & U^{*}(-U)^{-1} B+B^{*} 1 \\
0 * U^{-1}+1 * 0 & 0 *(-U)^{-1} B+1 * 1
\end{array}\right)=\left(\begin{array}{cc}
E_{n} & 0 \\
0 & 1
\end{array}\right) .
\end{gathered}
$$

Покажем теперь обратимость матрицы $U^{\prime}$. Вычислим произведение

$$
\begin{gathered}
P U^{\prime}=\left(\begin{array}{cc}
E_{n} & 0 \\
-C U^{-1} & 1
\end{array}\right)\left(\begin{array}{cc}
U & B \\
C & 1+C U^{-1} B
\end{array}\right)= \\
=\left(\begin{array}{cc}
E_{n} * U+0 * C & \left.E_{n} * B+0 * 1+C U^{-1} B\right) \\
\left(-C U^{-1}\right) * U+1 * C & \left(-C U^{-1}\right) * B+1 *\left(1+C U^{-1} B\right)
\end{array}\right)=\left(\begin{array}{cc}
U & B \\
0 & 1
\end{array}\right)=Q^{-1} .
\end{gathered}
$$

Теперь имеем равенства

$$
\left(P \cdot U^{\prime}\right) Q=Q^{-1} Q=E_{n+1} .
$$

Итак, $\left(P \cdot U^{\prime}\right) Q=E_{n+1}$ и $P, Q$ - обратимые матрицы в $K_{n+1}$. Тогда по лемме 2 $U^{\prime}$ - обратимая матрица и

$$
\begin{gathered}
\left(U^{\prime}\right)^{-1}=Q P=\left(\begin{array}{cc}
U^{-1} & (-U)^{-1} B \\
0 & 1
\end{array}\right)\left(\begin{array}{cc}
E_{n} & 0 \\
-C U^{-1} & 1
\end{array}\right)= \\
=\left(\begin{array}{cc}
U^{-1}+(-U)^{-1} B(-C) U^{-1} & (-U)^{-1} B \\
-C U^{-1} & 1
\end{array}\right) .
\end{gathered}
$$

Что и требовалось доказать. 
Теорема 4. Любая матрица из $K_{n}$ может быть записана как сумма диагональной и обратимой матриц.

Доказательство. Проведем индукцию по $n$. Так как $a=(a-1)+1$ для любого элемента $a$ из любого кольца $R$, то теорема верна для $n=1$.

Пусть она верна для кольца $K_{n}$, для некоторого $n \geq 1$. Покажем, что она верна и для кольца $K_{n+1}$.

Пусть $X \in K_{n+1}$. Напомним, что

$$
K_{n+1}=\left(\begin{array}{cccc}
R_{1} & M_{12} & \ldots & M_{1, n+1} \\
M_{21} & R_{2} & \ldots & M_{2, n+1} \\
\ldots & \ldots & \ldots & \ldots \\
M_{n+1,1} & M_{n+1,2} & \ldots & R_{n+1}
\end{array}\right),
$$

где $R_{1}, \ldots, R_{n+1}-$ некоторые кольца, $M_{i j}-R_{i}-R_{j}$-бимодуль, $i, j=1, \ldots, n+1$. Тогда можем записать

$$
X=\left(\begin{array}{ll}
A & B \\
C & d
\end{array}\right),
$$

где $A \in K_{n}, B$ - вектор-столбец длины $n, C$ - вектор-строка длины $n, d \in R_{n+1}$.

По предположению индукции $A$ - сумма диагональной и обратимой матриц, $A=D+U$, где $D-$ диагональная, $U-$ обратимая матрицы.

Тогда если положить

$$
\begin{gathered}
D^{\prime}=\left(\begin{array}{cc}
D & 0 \\
0 & d-1-C U^{-1} B
\end{array}\right), \\
U^{\prime}=\left(\begin{array}{cc}
U & B \\
C & 1+C U^{-1} B
\end{array}\right),
\end{gathered}
$$

то будет верно равенство $X=D^{\prime}+U^{\prime}$, где $D^{\prime}$ - диагональная матрица, $U^{\prime}$ - обратимая матрица (по лемме 3).

Что и требовалось доказать.

Таким образом, задача описания $k$-хороших колец обобщенных матриц сведена к задаче описания $(k-1)$-хороших диагональных матриц. В общем случае диагональная обобщенная матрица 2-хорошей не будет. Таким образом, встает вопрос - при каких условиях обеспечивается $k$-хорошесть диагональной обобщенной матрицы?

\section{ЛИТЕРАТУРА}

1. Крылов П.А., Туганбаев А.А. Модули над кольцами формальных матриц // Фундаментальная и прикладная математика. 2009. Т. 15. № 8. С. 145-211.

2. Крылов П.А. О группе $K_{0}$ кольца обобщенных матриц // Алгебра и логика. 2013. Т. 52. № 3. C. 370-385.

3. Tang G., Zhou Y. A class of formal matrix rings // Linear Algebra and Appl. 2013. V. 428. P. 4672-4688.

4. Wolfson K.G. An ideal theoretic characterization of the ring of all linear transformations // Amer. J. Math. 1953. V. 75. P. 358-386.

5. Zelinsky D. Every linear transformation is a sum of non-singular ones // Proc. Amer. Math. Soc. 1954. V. 5. P. 627-630.

6. Skornyakov L. Complemented modular lattices and regular rings. London: Oliver\&Boyd, 1958. $182 \mathrm{p}$. 
7. Raphael R.M. Rings which are generated by their units // J. Algebra. 1974. V. 28. P. 199204.

8. Fuchs L. Recent results and problems on Abelian groups // Topics in Abelian groups (Proc. Sympos., New Mexico State University). 1962. Scott, Foresman, Chicago. P. 9-40.

9. Stringall R.W. Endomorphism rings of Abelian groups generated by automorphism groups // Acta Math. Acad. Sci. Hungar. 1967. V. 18. P. 401-404.

10. Freedman H. On endomorphisms of primary Abelian groups // J. London Math. Soc. 1968. V. 43. P. 305-307.

11. Hill P. Endomorphism ring generated by units // Trans. Amer. Math. Soc. 1969. V. 141. P. 99-105.

12. Castagna F. Sums of automorphisms of a primary Abelian group // Pacific J. Math. 1968. V. 27. P. $463-473$.

13. Henriksen M. Two classes of rings generated by their units // J. Algebra. 1974. V. 31. P. 182193.

14. Vamos P. 2-good rings // Quart. J. Math. 2005. V. 56. P. 417-430.

15. Srivastava A.K. A survey of rings generated by units // Annales de la Faculte des Sciences de Toulouse Mathatiques. 2010. V. 19. P. 203-213.

Статья поступила 03.06.2015 г.

\section{Norbosambuev T.D. ON SUMS OF DIAGONAL AND INVERTIBLE FORMAL MATRICES}

\section{DOI $10.17223 / 19988621 / 36 / 4$}

This paper concerns properties of $k$-good formal matrix rings $K_{n}$ of order $n$ with rings $R_{1}, R_{2}$, $\ldots, R_{n}$ on the main diagonal and $R_{i}-R_{j}$-bimodules $M_{i j}$ on other places. In the ring theory, various matrix rings play an important role. Above all I mean formal matrix rings. Formal matrix rings generalize a notion of matrix ring of order $n$ over a given ring. Every ring with nontrivial idempotents is isomorphic to some formal matrix ring. The endomorphism ring of a decomposable module also is a formal matrix ring. The studies of such rings are quite useful for solving some problems on endomorphism rings of Abelian groups. In this paper I show that every matrix form $K_{n}$ is the sum of diagonal matrix and invertible matrix. Also I give one condition when $K_{n}$ is the $k$ good ring.

Keywords: ring, generalized matrix, formal matrix, $k$-good ring

NORBOSAMBUEV Tsyrendorji Dashatsyrenovich (Tomsk State University,

Tomsk, Russian Federation)

E-mail: NsTsdDts@yandex.ru

\section{REFERENCES}

1. Krylov P.A., Tuganbaev A.A. Moduli nad kol'tsami formal'nykh matrits. Fundamental'naya $i$ prikladnaya matematika, 2009, vol. 15, no. 8, pp. 145-211. (in Russian)

2. Krylov P.A. O gruppe $K_{0}$ kol'tsa obobshchennykh matrits. Algebra $i$ logika, 2013, vol. 52, no. 3, pp. 370-385. (in Russian)

3. Tang G., Zhou Y. A class of formal matrix rings. Linear Algebra and Appl., 2013, vol. 428, pp. 4672-4688.

4. Wolfson K.G. An ideal theoretic characterization of the ring of all linear transformations. Amer. J. Math., 1953, vol. 75, pp. 358-386.

5. Zelinsky D. Every linear transformation is a sum of non-singular ones. Proc. Amer. Math. Soc., 1954, vol. 5, pp. 627-630.

6. Skornyakov L. Complemented modular lattices and regular rings. London, Oliver\&Boyd, 1958. $182 \mathrm{p}$.

7. Raphael R.M. Rings which are generated by their units. J. Algebra, 1974, vol. 28, pp. 199204. 
8. Fuchs L. Recent results and problems on Abelian groups. Topics in Abelian groups (Proc. Sympos., New Mexico State University). Scott, Foresman, Chicago, 1962, pp. 9-40.

9. Stringall R.W. Endomorphism rings of Abelian groups generated by automorphism groups. Acta Math. Acad. Sci. Hungar., 1967, vol. 18, pp. 401-404.

10. Freedman H. On endomorphisms of primary Abelian groups. J. London Math. Soc., 1968, vol. 43, pp. 305-307.

11. Hill P. Endomorphism ring generated by units. Trans. Amer. Math. Soc., 1969, vol. 141, pp. 99-105.

12. Castagna F. Sums of automorphisms of a primary Abelian group. Pacific J. Math., 1968, vol. 27, pp. 463-473.

13. Henriksen M. Two classes of rings generated by their units. J. Algebra, 1974, vol. 31, pp. $182-193$.

14. Vamos P. 2-good rings. Quart. J. Math., 2005, vol. 56, pp. 417-430.

15. Srivastava A.K. A survey of rings generated by units. Annales de la Faculte des Sciences de Toulouse Mathatiques, 2010, vol. 19, pp. 203-213. 